\title{
In School-Age Children Who Were Born Very Preterm Sleep Efficiency Is Associated with Cognitive Function
}

\author{
Priska Hagmann-von Arx ${ }^{\mathrm{a}}$ Nadine Perkinson-Gloor ${ }^{\mathrm{a}}$ Serge Brand ${ }^{\mathrm{b}}$ \\ Djana Albert ${ }^{d}$ Edith Holsboer-Trachsler ${ }^{b}$ Alexander Grob ${ }^{a}$ Peter Weber ${ }^{c}$ \\ Sakari Lemola ${ }^{\text {a }}$ \\ ${ }^{a}$ Department of Psychology and ${ }^{b}$ Center for Affective, Stress and Sleep Disorders, Psychiatric University Clinics, \\ University of Basel, and 'Division of Neuropediatrics and Developmental Medicine, University Children's Hospital \\ Basel, Basel, and d University Clinic of Neurology, University Hospital of Bern, Bern, Switzerland
}

\section{Key Words}

Sleep · Polysomnography $\cdot$ Cognition · Prematurity .

School-age children

\begin{abstract}
Aims: This study examined whether the association between sleep duration, as well as sleep continuity, and cognitive function differs between normally developing preterm children compared to full-term children during middle childhood. Methods: A total of 58 early preterm ( $<32$ weeks' gestation) and 55 full-term children, aged 6-10 years and enrolled in elementary school, were assessed on sleep duration, sleep continuity and cognitive function. We used in-home polysomnographic recordings of total sleep time, sleep efficiency and nocturnal awakenings. Cognitive tests included intelligence, arithmetic, selective attention, verbal memory, and visuospatial memory. Results: Preterm children showed poorer performance in intelligence, arithmetic, selective attention, and visuospatial memory $(d=0.38-0.79, p<0.05)$
\end{abstract}

and more objectively assessed nocturnal awakenings $(\mathrm{d}=$ $0.40, p=0.03$ ) than full-term children. Associations of sleep efficiency and cognitive functions (intelligence, arithmetic, selective attention, visuospatial memory) were positive and stronger for preterm children $(\beta=0.17-0.31, p<0.05)$, while they were nonsignificant for full-term children. Conclusion: Results confirm lower cognitive test scores and more nocturnal awakenings in normally developing early preterm children compared to full-term children. Furthermore, poor sleep efficiency may aggravate cognitive deficits, particularly in children who are more vulnerable due to premature birth.

(c) 2015 S. Karger AG, Basel

\section{Introduction}

The incidence of very premature birth (birth before gestational age, GA $<32$ weeks) has been rising during the last two decades and the percentage of very prema-

\section{KARGER}

E-Mail karger@karger.com

www.karger.com/nps
(C) 2015 S. Karger AG, Basel

0302-282X/15/0704-0244\$39.50/0
Priska Hagmann-von Arx, PhD

Department of Psychology, University of Basel

Missionsstrasse 62

CH-4055 Basel (Switzerland)

E-Mail priska.hagmann@unibas.ch 
ture children surviving without major impairments has increased [1]. However, even among generally well-developing preterm children there is evidence of mild cognitive deficits [2-11]. One possible cause of mild cognitive deficits among preterm children involves subtle injuries to the cortical gray and white matter as well as secondary developmental disturbances of the brain [12].

Prematurely born children are also at risk of poor sleep, particularly due to sleep-disordered breathing (SDB) [13]. Generally, an adequate amount of good-quality sleep is considered important for children's development [14]. In healthy children, sleep duration and sleep efficiency (defined as the proportion of the total amount of time spent in bed that the child is asleep) have been particularly well studied with regard to their relation with cognitive functions [15], covering a wide variety of both experimental $[16,17]$ and correlational [18] studies. A recent meta-analysis [15] including studies with more than 30,000 healthy children showed that sleep duration is positively associated with cognitive function, with effect sizes in the modest range. With regard to objectively assessed sleep continuity (which includes sleep efficiency, sleep latency, time awake after sleep onset, and number of awakenings [19]) sleep efficiency, in particular, was positively related to visual and auditory working memory [20], cognitive processing speed $[21,22]$ and abstract reasoning [18], although results were not very consistent across studies [15]. Moreover, findings indicate that some children may be more vulnerable to the effects of poor sleep efficiency than others. One study, for instance, found a positive relation between sleep efficiency and working memory only in children from poor socioeconomic status, who may be considered more at risk of poor outcomes [21].

The current understanding of the association between sleep and cognitive functions in preterm children is limited. One in-home overnight cardiorespiratory study [23] showed that preterm children with SDB achieved significantly lower vocabulary and achievement test scores relative to preterm children without SDB, while there were no such differences between full-term children with and without SDB. These results indicate that the strength of the relationship between sleep and cognitive functions may vary between children depending on their prematurity status, such that preterm children are particularly vulnerable to the detrimental effects of poor sleep. Thus, prematurity status may be a factor that makes children more vulnerable to the effects of poor sleep. These findings also appear consistent with the cognitive reserve hy- pothesis which claims that individuals differ in cognitive vulnerability to stressors such as poor sleep [24].

To our knowledge, no study has examined the moderating role of prematurity status in the relationship between objectively assessed sleep duration and sleep continuity using polysomnography and cognitive functions. The main goal of the present study was to fill this gap by assessing cognitive functions in very preterm and fullterm children during middle childhood in relation to objectively assessed sleep duration and sleep efficiency, which are the two most widely studied objective sleep variables in pediatric sleep research [15]. Moreover, with regard to sleep continuity, we also studied the number of nocturnal awakenings (number of arousals from sleep), which we expected to be particularly related to prematurity status, as previous research has shown that prematurity is a risk factor for developing SDB [13].

We tested the following hypotheses. First, we hypothesized to find greater deficits in cognitive functions, shorter sleep duration and poorer sleep continuity (lower sleep efficiency and more nocturnal awakenings) in preterm than full-term children. Second, we hypothesized to find a stronger association between sleep duration/sleep continuity and cognitive functions in preterm than fullterm children.

\section{Methods}

\section{Subjects}

Between June 2011 and June 2012, 58 preterm and 55 full-term children were enrolled in the study. The sample of preterm children was derived from an initial cohort of 217 preterm children born in Basel, Switzerland and treated between June 2001 and December 2005 at the University Children's Hospital Basel. Hospital treatment records were examined with regard to children's prematurity (defined as GA $<32$ weeks at birth). Children with serious conditions such as severe developmental delay at age 2 , children without information on development until age 2 due to aftercare at a different hospital or due to early death and children of parents with insufficient German language skills to give informed consent were excluded from the recruitment process. Of the remaining 133 children, $21 \%$ had moved from the area and $11 \%$ could not be contacted due to no or incorrect address information. Finally, 90 children could be contacted, of whom 58 (64\%) agreed to participate. There were no differences between participating preterm children and nonparticipants with regard to birth weight (mean $=1,302$ vs. $\left.1,284 \mathrm{~g}, \mathrm{~F}_{1,216}=0.09, \mathrm{p}=0.76\right)$, $\mathrm{GA}($ mean $=29.7$ vs. 29.7 weeks at birth, $\left.\mathrm{F}_{1,216}=0.003, \mathrm{p}=0.96\right)$ and treatment duration at the neonatal intensive care unit (mean $=53.9$ vs. 53.1 days, $\mathrm{F}_{1,180}=0.048, \mathrm{p}=0.83$ ). Demographic characteristics are presented in table 1 . The sample of preterm children included 13 extremely preterm children (GA $<28$ weeks at birth, mean GA 26.8 weeks, 8 boys, 5 girls, mean age 8.3 years) and 45 very preterm 
Table 1. Demographic characteristics of preterm and full-term children

\begin{tabular}{|c|c|c|c|c|c|c|c|}
\hline & \multicolumn{3}{|l|}{ Preterm } & \multirow{2}{*}{$\begin{array}{l}\text { Full-term } \\
\mathrm{D} \\
\text { control } \\
(\mathrm{n}=55)\end{array}$} & \multicolumn{2}{|c|}{ A vs. B vs. D } & \multirow{2}{*}{$\frac{C \text { vs. } D}{\text { p value }}$} \\
\hline & $\begin{array}{l}\text { A } \\
\text { GA 25-27 } \\
\text { weeks }(n=13)\end{array}$ & $\begin{array}{l}\text { B } \\
\text { GA 28-31 } \\
\text { weeks }(n=45)\end{array}$ & $\begin{array}{l}\text { C } \\
\text { total } \\
(n=58)\end{array}$ & & $\mathrm{p}$ value & $\begin{array}{l}\text { group } \\
\text { differences }\end{array}$ & \\
\hline Age, years & $8.3 \pm 1.3$ & $8.2 \pm 1.3$ & $8.2 \pm 1.3$ & $8.3 \pm 1.3$ & 0.819 & & 0.530 \\
\hline Male & $8(62)$ & $32(71)$ & $40(69)$ & $35(64)$ & 0.679 & & 0.549 \\
\hline Infant respiratory distress syndrome & $12(92)$ & $33(73)$ & $45(78)$ & $0(0)$ & 0.136 & & $<0.001$ \\
\hline Apnea of prematurity & $11(85)$ & $32(71)$ & $46(79)$ & $0(0)$ & 0.480 & & $<0.001$ \\
\hline BMI, $\mathrm{kg} / \mathrm{m}^{2}$ & $17.1 \pm 3.1$ & $16.1 \pm 2.4$ & $16.3 \pm 2.2$ & $16.9 \pm 1.9$ & 0.189 & & 0.213 \\
\hline Mother tongue (German) & $4(31)$ & $34(76)$ & $38(66)$ & $42(76)$ & 0.005 & $\mathrm{BD}>\mathrm{A}$ & 0.205 \\
\hline Maternal education & & & & & 0.001 & $\mathrm{D}>\mathrm{AB}$ & $<0.001$ \\
\hline No vocational education & $3(23)$ & $6(13)$ & $9(16)$ & $3(6)$ & & & \\
\hline
\end{tabular}

Data are presented as means \pm SD or $\mathrm{n}(\%)$. p values: $\chi^{2}$ test, Fisher's exact test or analyses of variance.

children (GA 28-31 weeks at birth, mean GA 30.5 weeks, 32 boys, 13 girls, mean age 8.2 years). None of the children suffered from periventricular leukomalacia, while 4 out of the 58 preterm children were diagnosed with mild intraventricular hemorrhage (IVH) - 1 child had IVH grade 1 and 3 children had IVH grade 2 .

A total of 55 control children born after the 37 th week of gestation ( 35 boys, 20 girls, mean GA 39.7 weeks, mean age 8.3 years) were recruited from birth announcements in newspapers. All children attended primary school in Switzerland. The samples were comparable regarding age and sex. As the parents of some of the full-term children refused participation, we were unable to recruit controls for 3 of the 58 preterm children. The Ethics Committee of Basel approved the study protocol. Parents gave written informed consent for the children to participate and assent was obtained from the child prior to the beginning of the study.

\section{Procedure and Measures}

All children were visited at home on a regular school day at late afternoon by trained study personnel. The study used cognitive test procedures and in-home polysomnography, as well as other physiological and behavioral assessments which are not presented here.

Cognitive tests were administered in a quiet room. A short form of the German version of the Wechsler Intelligence Scale for Children, fourth edition (WISC-IV) [25], including the subtests vocabulary, matrix reasoning, letter-number sequencing, and coding, was used to estimate the children's intelligence (IQ). Children's IQ scores were obtained based on the four subtests using weighted calculation, following the procedure proposed by Waldmann [26]. The IQ has a mean of $100($ SD = 15). Arithmetic was administered as a supplemental subtest, which is standardized with a mean of $10(\mathrm{SD}=3)$. Selective attention was measured using the selective attention subtest of the Intelligence and Development Scales (IDS) [27, 28], where the children were asked to find and mark drawings of ducks with specific attributes in lines of different ducks within a stated time. This measure has a mean of 10 (SD = 3). Verbal memory was measured using the German version of the Rey Auditory Verbal Learning Test (AVLT) [29]. The children were given 5 trials to learn a list of 15 words, which they were asked to recall after each trial (learning). An interference trial of a different word list was presented, followed by a 6th recall trial of the original word list (postinterference test). Finally, after a 30 -min interval the children were asked to identify the original word list out of 50 words (recognition). These measures have $t$ scores with a mean of $50(\mathrm{SD}=10)$. Visuospatial memory was measured using the Rey-Osterrieth Complex Figure test (ROCF) [30]. A complex geometric figure was presented to the children, which they had to copy and reproduce from memory $30 \mathrm{~min}$ later (delayed recall). Both subtests have a score range of $0-36$. A small number of test scores were missing due to child noncompliance, equipment failure or restrictions in testing time (preterm children: WISC-IV, $\mathrm{n}=$ 3 ; IDS, $\mathrm{n}=3$; AVLT, $\mathrm{n}=1$; ROCF, $\mathrm{n}=2$; full-term children: WISCIV, $\mathrm{n}=2$; ROCF, $\mathrm{n}=3$ ).

To assess sleep duration and sleep continuity, overnight polysomnographic assessment of sleep was performed at the children's homes during the night following cognitive testing using the Somté PSG (Compumedics, Singen, Germany) portable sleep-monitoring device. EEG, electrooculography, and electromyography were assessed. Electrodes for EEG recordings were placed at C3 and C4 (with cross-reference to A2 and A1) and sleep scoring was performed in 30-second epochs according to standard procedures [31]. The following indices of sleep duration and sleep continuity were derived: total sleep time (time in bed minus time spent awake), sleep efficiency (the ratio of total sleep time to time in bed) and nocturnal awakenings (number of arousals from sleep). Indices of sleep architecture or sleep microarchitecture are not reported here as their relationship to cognitive function is beyond the scope of the present study. Sleep scoring was conducted by two experienced sleep technologists. Because 
Table 2. Distribution of cognitive functions and sleep measures in preterm and full-term children

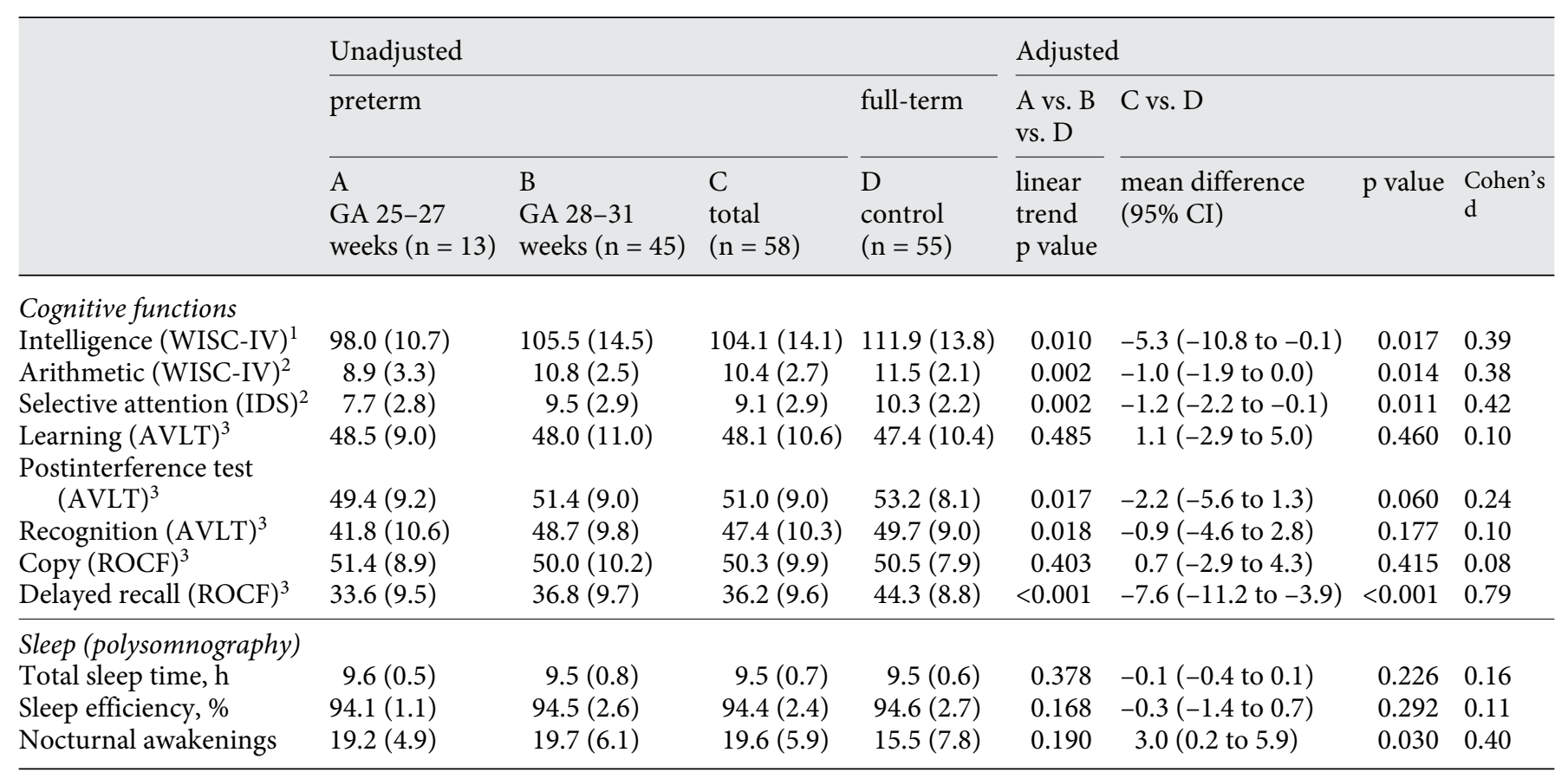

Adjusted analyses controlled for mother tongue, maternal education and (for sleep variables) child age.

${ }^{1}$ IQ score normative mean $=100(S D=15) .{ }^{2}$ Scaled score normative mean $=10(\mathrm{SD}=3) .{ }^{3}$ t score normative mean $=50(\mathrm{SD}=10)$.

of technical error during the measurement period, polysomnographic data were not available for 6 preterm children and 5 fullterm children.

\section{Statistical Analyses}

To test for group differences in demographic characteristics, $\chi^{2}$ analyses or Fisher's exact tests were used for categorical variables and analyses of variance were used for continuous variables. Due to possible associations of cognitive variables with maternal education [32] and migration background [33], we included maternal education and mother tongue (as an indicator of migration background) in the analyses of the group differences. Results showed both variables to be associated with prematurity status and, therefore, they were included a priori as covariates in the following group comparisons. Analyses of covariance tested whether the groups differed significantly in sleep variables and in cognitive functions. Tests for linear trend were conducted to test for increasing or decreasing sleep scores and cognitive performance across groups (extremely preterm, very preterm, full-term). For analyses of covariance (mean differences between groups), the reported $p$ values are one-tailed, given our directional hypothesized predictions. Additionally, effect sizes (Cohen's d) are reported. If an extreme value (defined as a $\mathrm{z}$-score exceeding 3 SDs from the group mean) occurred in the sleep variables $(n=3)$, scores were truncated to \pm 3 SD. Hierarchical regression analyses were conducted following the procedure proposed by Aiken and West [34] to analyze the associations between sleep and cognitive functions and to investigate whether prematurity status (preterm, full-term) acts as a moderator of these associations. Demographic characteristics, including maternal education (three-level categorical variable), mother tongue, age, and sex, were entered in the first block. Prematurity status and sleep variables were entered in the next block and, finally, the two-way interaction terms between prematurity status and sleep variables were entered in the last block. Raw scores of each variable were z-transformed across both groups. For moderated hierarchical regression models, adjusted t values, standardized betas and adjusted $\mathrm{p}$ values (two-tailed) are reported. If a significant interaction was found (indicating a significant moderation effect), then the interaction was graphed by computing predicted values of cognitive functions separately for preterm and full-term children at low (-1 SD) and high (+1 SD) values of sleep variables. Separate multiple regression analyses were used to evaluate whether the slopes in the graphs were significantly different from zero [34]. For separate slope analyses reported $p$ values are one-tailed.

\section{Results}

\section{Cognitive Functions and Sleep in Preterm and Full-Term Children}

The results of the cognitive functions and the mean sleep scores are presented in table 2 . The performance of preterm children in tests of cognitive functions was in the 
average range except for ROCF delayed recall, where the mean score for the preterm group $($ mean $=36.2, \mathrm{SD}=9.6)$ was well below the normative mean. Adjusting for maternal education and mother tongue, preterm children reached significantly lower scores than full-term children in IQ, arithmetic, selective attention, and visuospatial memory (ROCF delayed recall), whereas the linear trend was additionally significant for verbal memory (AVLT postinterference test, recognition), indicating systematically increasing test scores from extremely preterm children to full-term children. No significant differences were found for AVLT learning and ROCF copy. With regard to sleep, preterm children showed more nocturnal awakenings than full-term children, controlling for maternal education, mother tongue and child age. No significant differences were found for sleep duration or sleep efficiency, which was on a relatively high average level of more than $94 \%$ in both groups. These findings did not change substantially when the 4 preterm children with mild IVH were excluded from the analyses (data not shown).

\section{Associations between Sleep and Cognitive Functions}

First, hierarchical regression analyses were calculated for the combined participant sample. Controlling for maternal education, mother tongue, child age, sex, and prematurity status, better sleep efficiency was significantly associated with higher scores in the verbal memory postinterference test of the AVLT $(\mathrm{t}=2.537, \beta=0.245, \mathrm{p}=$ $0.013)$. Other sleep variables showed no association with cognitive performance.

Second, moderated hierarchical regression analyses were calculated with prematurity status $\times$ sleep interaction terms, controlling for maternal education, mother tongue, child age, sex, prematurity status, and the corresponding sleep variable (table 3 ). These analyses revealed four significant prematurity status $\times$ sleep interactions. Prematurity status moderated the association between sleep efficiency and IQ $(\mathrm{t}=2.015, \beta=0.145, \mathrm{p}=0.047$, $\left.\Delta \mathrm{R}^{2}=0.018\right)$, arithmetic $(\mathrm{t}=2.068, \beta=0.121, \mathrm{p}=0.042$, $\left.\Delta \mathrm{R}^{2}=0.012\right)$, selective attention $(\mathrm{t}=2.158, \beta=0.183$, $\mathrm{p}=$ $0.034, \Delta \mathrm{R}^{2}=0.026$ ), and visuospatial memory (ROCF copy: $\mathrm{t}=2.623, \beta=0.269, \mathrm{p}=0.010, \Delta \mathrm{R}^{2}=0.061$ ).

As depicted in figure 1 , in preterm children, sleep efficiency was associated with performance in IQ $(t=1.729$, $\beta=0.166, \mathrm{p}=0.045)$, arithmetic $(\mathrm{t}=1.880, \beta=0.169, \mathrm{p}=$ $0.033)$, selective attention $(t=1.776, \beta=0.204, p=0.041)$, and visuospatial memory (ROCF copy: $t=2.257, \beta=$ $0.311, p=0.015$ ), such that better sleep efficiency was related with better performance, whereas in full-term children there were no associations between sleep efficiency and IQ $(\mathrm{t}=-0.982, \beta=-0.118, \mathrm{p}=0.166)$, arithmetic $(\mathrm{t}=$ $-0.950, \beta=-0.078, p=0.174)$, selective attention $(t=$ $-0.734, \beta=-0.091, p=0.233$ ), and visuospatial memory (ROCF copy: $\mathrm{t}=-0.900, \beta=-0.149, \mathrm{p}=0.186$ ).

These findings did not change substantially when the extremely preterm children or the 4 preterm children with mild IVH were excluded from the analyses or when additionally controlling for total sleep time or nocturnal awakenings (data not shown).

\section{Discussion}

In our study, preterm children scored mainly in the average range but achieved lower IQ scores and fared worse in arithmetic, selective attention and visuospatial memory relative to full-term children, controlling for maternal education and mother tongue. The effect sizes of these differences were in the small-to-medium range. Lower test scores in IQ, arithmetic, selective attention, verbal memory (postinterference test, recognition), and visuospatial memory (delayed recall) were related to the degree of prematurity, such that there were systematic increases in test scores from extremely preterm to fullterm children. By contrast, group differences in the verbal memory learning subtest and in the visuospatial memory copy subtest did not reach significance. With regard to sleep, preterm children showed more nocturnal awakenings compared to full-term children, while no group differences were found for sleep duration and sleep efficiency. Furthermore, our findings were consistent with the hypothesis that the associations of sleep and cognitive function were moderated by prematurity status. Compared to full-term children, in preterm children sleep efficiency was more strongly related to performance in IQ, arithmetic, selective attention, and visuospatial memory (copy), such that better sleep efficiency was associated with better performance. No moderation was found for sleep duration and nocturnal awakenings.

Our results are generally consistent with previous research showing lower IQ scores $[5,35]$ and more selective attention problems $[5,6,9]$, as well as poorer performance in mathematics $[36,37]$ and visuospatial memory [2], in preterm children compared to full-term children. The finding that preterm children achieved lower scores in two out of three verbal memory subtests is in line with previous results on verbal memory showing deficits in preterm children $[5,6]$, although in another study there were no such group differences [10]. The finding that lower cognitive test scores are in relation to the degree of 
Table 3. Hierarchical regressions with sleep measures $\times$ prematurity status interactions predicting cognitive functions

\begin{tabular}{|c|c|c|c|c|c|c|c|c|}
\hline \multicolumn{9}{|l|}{ Step 1} \\
\hline \multicolumn{9}{|l|}{ Maternal education: } \\
\hline No vocational education & $-0.289^{* *}$ & -0.128 & -0.136 & -0.038 & -0.122 & $-0.333^{* *}$ & $-0.273^{*}$ & -0.127 \\
\hline Sex & 0.081 & -0.045 & -0.047 & $0.249^{* *}$ & 0.028 & 0.100 & 0.052 & 0.059 \\
\hline Age & $0.705^{* * *}$ & $0.805^{* * *}$ & $0.565^{* * *}$ & $0.491^{* * *}$ & $0.395^{* * *}$ & $0.305^{* * *}$ & $0.277^{* *}$ & $0.315^{* * *}$ \\
\hline Prematurity status & $-0.143^{*}$ & -0.107 & $-0.226^{* *}$ & 0.033 & -0.136 & -0.078 & 0.024 & $-0.359^{* * *}$ \\
\hline \multicolumn{9}{|l|}{ Step $2 a$} \\
\hline Total sleep time & 0.081 & 0.021 & 0.113 & 0.149 & 0.169 & 0.140 & 0.107 & 0.092 \\
\hline Prematurity status $\times$ total sleep time & 0.055 & 0.092 & 0.109 & 0.132 & 0.127 & 0.101 & 0.061 & -0.023 \\
\hline Sleep efficiency & 0.058 & 0.066 & 0.109 & 0.178 & $0.241^{*}$ & 0.147 & 0.199 & 0.140 \\
\hline Prematurity status $\times$ sleep efficiency & $0.145^{*}$ & $0.121^{*}$ & $0.183^{*}$ & 0.120 & 0.176 & 0.090 & $0.269^{*}$ & 0.193 \\
\hline $\mathrm{F}$ change of interaction & $4.058^{*}$ & $4.278^{*}$ & $4.657^{*}$ & 1.720 & 3.493 & 0.946 & $6.880^{*}$ & 3.922 \\
\hline F of total model & $17.699^{* * *}$ & $32.376^{* * *}$ & $11.000^{* * *}$ & $4.932^{* * *}$ & $3.874^{* *}$ & $4.294^{*}$ & $3.138^{* *}$ & $4.639^{* * *}$ \\
\hline \multicolumn{9}{|l|}{ Step $2 c$} \\
\hline Nocturnal awakenings & 0.071 & -0.019 & 0.060 & 0.011 & 0.117 & 0.037 & 0.092 & -0.068 \\
\hline \multicolumn{9}{|l|}{ Prematurity status $\times$ nocturnal } \\
\hline awakenings & -0.061 & 0.015 & -0.005 & -0.012 & 0.001 & 0.072 & -0.036 & -0.030 \\
\hline F change of interaction & 0.786 & 0.071 & 0.003 & 0.018 & 0.000 & 0.590 & 0.123 & 0.099 \\
\hline F of total model & $16.956^{* * *}$ & $30.224^{* * *}$ & $9.985^{* * *}$ & $4.147^{* * *}$ & $2.729^{*}$ & $3.913^{* *}$ & $2.051^{*}$ & $3.920^{* *}$ \\
\hline
\end{tabular}

Coefficients are standardized regression coefficients unless otherwise indicated. Step 1: model with control variables predicting cognitive function. Step 2: model with sleep variables and prematurity status $\times$ sleep interaction terms predicting cognitive function, controlled for variables entered in step 1 . Maternal education: vocational education served as reference category. Mother tongue: $+1=$ foreign language, $-1=$ German language. Sex: $+1=$ girl; $-1=$ boy . Prematurity status: $+1=$ preterm birth, $-1=$ full-term birth. Age: shows strong positive associations with cognitive functions as the analyses were conducted with $\mathrm{z}$-standardized raw scores of cognitive measures. ${ }^{*} \mathrm{p}<0.050{ }^{* *} \mathrm{p}<0.010$; ${ }^{* *} \mathrm{p}<0.001$.

prematurity has been reported in previous research $[1,4]$. Nevertheless, except for one task of visuospatial memory (delayed recall), the majority of the preterm children performed within the average range and the group differences between preterm and full-term children resulted because of the high average performance of the controls. The finding that preterm children showed more nocturnal awakenings than full-term children corresponds with research showing more disturbed sleep due to SDB in preterm children [13], as obstructed respiration may result in arousals from sleep.

Apart from one significant association between sleep efficiency and the verbal memory postinterference subtest, we found no direct relationship of sleep duration and sleep continuity with cognitive function. While this is in conflict with previous research $[15,18,20-22]$, it is in line with the notion that not all children are equally vulnerable to the effect of poor sleep [20]. Our findings are also in line with the results from Emancipator et al. [23], who reported that preterm children with objectively assessed SDB had significantly lower cognitive test scores relative to preterm children without SDB, whereas there were no significant differences in cognitive test scores between full-term children with and without SDB. The evidence from our study as well as from Emancipator et al. [23] indicates an increased vulnerability of preterm children to the impairing effects of sleep disruptions on cognitive functions. From the perspective of the cognitive reserve hypothesis [24], one may expect that preterm children are less able than full-term children to compensate for stressors such as disturbed sleep, possibly due to subtle neonatal brain injury $[24,38,39]$. Thus, 
Fig. 1. Sleep variables $\times$ prematurity status interactions in prediction of cognitive functions. For slopes that were significantly different from zero, the $\mathrm{p}$ value is presented next to the slope.

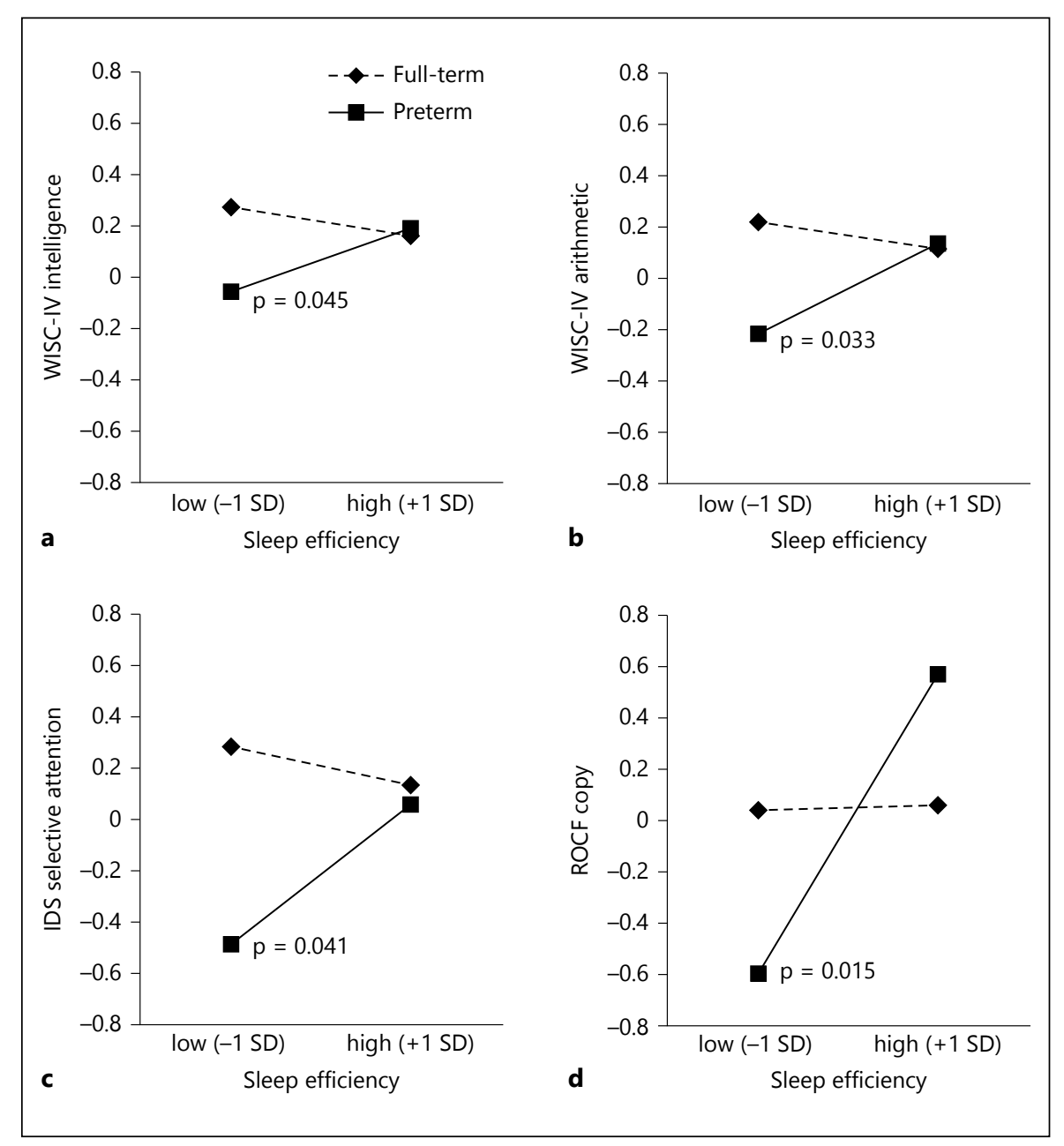

prematurity may be a factor increasing the vulnerability to the impairing effects of poor sleep. There is evidence of individual differences in vulnerability to sleep deprivation, which appear to be mediated by differential cortical activation patterns when acutely sleep deprived [24, 40]. Future studies may examine whether brain activity differs in full-term and preterm children according to their sleep quality. Another research approach may be taken to examine whether the family environment (and therefore possibly also the sleep environment) of children with lower cognitive ability is more disruptive. For instance, it is known that children in a less secure family environment more often show both worse sleep pattern and decreased academic and behavioral functioning compared to children growing up in a warm and supportive family environment $[41,42]$. Thus, it is possible that variables that have not been assessed in the current study such as family functioning or the parent-child re- lationship have an influence on both sleep pattern and cognitive function.

Conflicting with previous research, the present study shows no significant differences between preterm and full-term children in the visuospatial memory copy subtest, even though a previous study has suggested that preterm children would be particularly impaired in this task [2].

Differences with previous research may be seen in the light that we studied a generally healthy sample of preterm children. Not all parents of preterm children agreed to participate, which may have led to a selection bias towards more healthily developing preterm children, as it is possible that parents may tend to avoid situations where their child's deficits are highlighted [43].

Limitations of the study include, firstly, that only one night of polysomnography was available, while assessment of sleep during further nights might have increased 
the reliability of sleep variables and, secondly, that no cardiorespiratory assessment of SDB was conducted. By contrast, we consider it a strength that sleep variables were assessed using in-home instead of laboratory-based polysomnography to allow children to sleep in their familiar environment. Finally, the correlative design of the study precludes causal inferences. It does not allow testing the direction of the effects between sleep and cognitive functions. Moreover, it remains possible that the here-reported associations were due to unobserved confounding variables.

In conclusion, the results indicate that preterm children who are enrolled in normal school remain at higher risk for cognitive deficits and sleep disturbances. Further, the results of our study are consistent with the view that preterm children are particularly vulnerable to the impairing effects of poor sleep efficiency for cognitive function.

\section{Acknowledgments}

This research was financially supported by the Swiss National Science Foundation (project: sleep, cognitive, and socio-emotional development in preterm children during middle and late childhood; grant No: 143962) and the Research Fund of the University of Basel. We thank Marielle Koenig and Vladimir Djurdjevic, Center for Affective, Stress and Sleep Disorders, Psychiatric University Clinics of the University of Basel, Basel, Switzerland, for scoring polysomnographic data.

\section{References}

1 Saigal S, Doyle LW: An overview of mortality and sequelae of preterm birth from infancy to adulthood. Lancet 2008;371:261-269.

2 Anderson PJ, Doyle LW: Executive functioning in school-aged children who were born very preterm or with extremely low birth weight in the 1990s. Pediatrics 2004;114:5057.

-3 Hutchinson EA, De Luca CR, Doyle LW, Roberts G, Anderson PJ: School-age outcomes of extremely preterm or extremely low birth weight children. Pediatrics 2013;131:10531061.

4 Marlow N, Wolke D, Bracewell MA, Samara M: Neurologic and developmental disability at six years of age after extremely preterm birth. N Engl J Med 2005;352:9-19.

5 Mikkola K, Ritari N, Tommiska V, Salokorpi $\mathrm{T}$, Lehtonen L, Tammela O, Pääkönen L, Olsen P, Korkman M, Fellman V: Neurodevelopmental outcome at 5 years of age of a national cohort of extremely low birth weight infants who were born in 1996-1997. Pediatrics 2005;116:1391-1400.

-6 Orchinik LJ, Taylor HG, Espy KA, Minich N, Klein N, Sheffield T, Hack M: Cognitive outcomes for extremely preterm/extremely low birth weight children in kindergarten. J Int Neuropsychol Soc 2011;17:1067-1079.

7 Saigal S, den Ouden L, Wolke D, Hoult L, Paneth N, Streiner DL, Whitaker A, Pinto-Martin J: School-age outcome in children who were extremely low birth weight from four international population-based cohorts. Pediatrics 2003;112:943-950.

-8 Aarnoudse-Moens CSH, Smidts DP, Oosterlaan J, Duivenvoorden HJ, Weisglas-Kuperus $\mathrm{N}$ : Executive function in very preterm children at early school age. J Abnorm Child Psychol 2009;37:981-993.

$\checkmark 9$ Bayless S, Stevenson J: Executive functions in school-age children born very prematurely. Early Hum Dev 2007;83:247-254.
10 Lind A, Korkman M, Lehtonen L, Lapinleimu H, Parkkola R, Matomäki J, Haataja L: The Pipari Study Group: Cognitive and neuropsychological outcomes at 5 years of age in preterm children born in the 2000s. Dev Med Child Neurol 2011;53:256-262.

11 Lemola S: Long-term outcomes of very preterm birth: mechanisms and interventions. Eur Psychol 2014, in press.

12 Volpe JJ: Brain injury in premature infants: a complex amalgam of destructive and developmental disturbances. Lancet Neurol 2009; 8:110-124

13 Rosen CL, Larkin EK, Kirchner HL, Emancipator JL, Bivins SF, Surovec SA, Martin RJ, Redline S: Prevalence and risk factors for sleep-disordered breathing in 8- to 11-yearold children: association with race and prematurity. J Pediatr 2003;142:383-389.

14 Dahl RE: The impact of inadequate sleep on children's daytime cognitive function. Semin Pediatr Neurol 1996;3:44-50.

15 Astill RG, Van der Heijden KB, Van IJzendoorn MH, Van Someren EJ: Sleep, cognition, and behavioral problems in school-age children: a century of research meta-analyzed. Psychol Bull 2012;138:1109-1138.

16 Fallone G, Acebo C, Arnedt JT, Seifer R, Carskadon MA: Effects of acute sleep restriction on behavior, sustained attention, and response inhibition in children. Percept Mot Skills 2001;93:213-229.

17 Sadeh A, Gruber R, Raviv A: The effects of sleep restriction and extension on school-age children: what a difference an hour makes. Child Dev 2003;74:444-455.

18 Paavonen EJ, Räikkönen K, Pesonen AK, Lahti J, Komsi N, Heinonen K, Järvenpää AL, Strandberg T, Kajantie E, Porkka-Heiskanen T: Sleep quality and cognitive performance in 8-yearold children. Sleep Med 2010;11:386-392.

19 Perlis ML, Gehrman P, Thase ME: Mood disorders and sleep; in Kushida C (ed): Encyclo- pedia of Sleep. Waltham, Academic Press, 2002, vol 2, pp 380-384.

20 Steenari MR, Vuontela V, Paavonen EJ, Carlson S, Fjallberg M, Aronen E: Working memory and sleep in 6- to 13-year-old school children. J Am Acad Child Adolesc Psychiatry 2003;42:85-92.

21 Buckhalt JA, El-Sheikh M, Keller P: Children's sleep and cognitive functioning: race and socioeconomic status as moderators of effects. Child Dev 2007;78:213-231.

22 Sadeh A, Gruber R, Raviv A: Sleep, neurobehavioral functioning, and behavior problems in school-age children. Child Dev 2002;73: 405-417.

23 Emancipator JL, Storfer-Isser A, Taylor HG, Rosen CL, Kirchner HL, Johnson NL, Zambito AM, Redline S: Variation of cognition and achievement with sleep-disordered breathing in full-term and preterm children. Arch Pediatr Adolesc Med 2006;160:203210.

24 Goel N, Rao H, Durmer JS, Dinges DF: Neurocognitive consequences of sleep deprivation. Semin Neurol 2009;29:320-339.

25 Petermann F, Petermann U: Wechsler Intelligence Scale for Children (WISC-IV). Frankfurt, Pearson Assessment, 2011.

26 Waldmann HC: German WISC-IV short forms: a scenario-based evaluation of statistical properties. Diagnostica 2008;54:202210

27 Grob A, Meyer CS, Hagmann-von Arx P: Intelligence and Development Scales. Bern, Hans Huber, 2009.

28 Hagmann-von Arx P, Grob A, Petermann F, Daseking M: Concurrent validity of the HAWIK-IV and the Intelligence and Development Scales (IDS). Z Kinder Jugendpsychiatr Psychother 2012;40:41-50.

29 Helmstaedter C, Lendt M, Lux S: Verbaler Lern- und Merkfähigkeitstest. Göttingen, Beltz, 2001. 
30 Knight J, Kaplan E: The handbook of Rey-Osterrieth Complex Figure Usage: Clinical and Research Applications. Lutz, Psychological Assessment Resources, 2003.

31 Rechtschaffen A, Kales A: A manual for standardized terminology, techniques and scoring system for sleep stages of human subjects; in Brain Information Service (NIH Publ No 204). Los Angeles, Brain Research Institute, 1968.

-32 Bradley RH, Corwyn RF: Socioeconomic status and child development. Annu Rev Psychol 2002;53:371-399.

33 Hagmann-von Arx P, Petermann F, Grob A: Convergent and discriminant validity of the WISC-IV and the Intelligence and Development Scales (IDS) in children with a migration background. Diagnostica 2013;59:170182.
34 Aiken LS, West SG: Multiple Regression: Testing and Interpreting Interactions. London, Sage, 1991.

35 Bhutta AT, Cleves MA, Casey PH, Cradock MM, Anand KJS: Cognitive and behavioral outcomes of school-aged children who were born preterm. JAMA 2002;288:728-737.

36 Aarnoudse-Moens CSH, Weisglas-Kuperus N, van Goudoever JB, Oosterlaan J: Metaanalysis of neurobehavioral outcomes in very preterm and/or very low birth weight children. Pediatrics 2009;124:717-728.

37 Botting N, Powls A, Cooke R, Marlow N: Cognitive and educational outcome of very-low birthweight children in early adolescence. Dev Med Child Neurol 2008;40:652-660.

38 Kesler SR, Ment LR, Vohr B, Pajot SK, Schneider KC, Katz KH, Ebbitt TB, Duncan CC, Makuch RW, Reiss AL: Volumetric analysis of regional cerebral development in preterm children. Pediatr Neurol 2004;31:318-325.
39 Luciana M: Cognitive development in children born preterm: implications for theories of brain plasticity following early injury. Dev Psychopathol 2003;15:1017-1047.

40 Basner M, Rao H, Goel N, Dinges DF: Sleep deprivation and neurobehavioral dynamics. Curr Op Neurobiol 2013;23:854-863.

41 El-Sheikh M, Buckhalt JA, Keller PS, Cummings EM, Acebo C: Child emotional insecurity and academic achievement: the role of sleep disruptions. J Fam Psychology 2007;21: 29-38.

42 El-Sheikh M, Buckhalt JA, Cummings EM, Keller P: Sleep disruptions and emotional insecurity are pathways of risk for children. J Child Psychol Psychiatry 2007;48:88-96.

43 Wolke D, Söhne B, Ohrt B, Riegel K: Followup of preterm children: important to document dropouts. Lancet 1995;345:447. 